\title{
Comment on: The Factors Associated with Carpal Tunnel Syndrome Severity
}

\author{
Yousef FALLAH, Behnam BAGHIANIMOGHADAM \\ Department of Orthopedic Surgery, Tehran University of Medical Sciences, Tehran, Iran \\ Corresponding author: Behnam BAGHIANIMOGHADAM behnam.baghian@gmail.com
}

\section{Dear editor in chief}

W e have read the recently published article by Tonga and Bahadir with great interest (2). In another valuable recent study in this area, which is not discussed in the Tonga and Bahadir's article, Rhee et al. used a national database to examine carpal tunnel syndrome (CTS) risk factors (1). They also used electrodiagnosis to confirm CTS. Patients were followed in this study for 11 years and compared to the control group. They found no significant differences between diabetes, gout, and end-stage renal disease (ESRD) with CTS, but found a significant association between rheumatoid arthritis, hypothyroidism, and Raynaud's syndrome with CTS. They also found that women had a 4.13 hazard ratio for CTS. Hazard ratios for CTS also increased with the increase in BMI. Hazard ratios for RA and hypothyroidism were 1.93 and 1.61 , respectively. These results are similar to the Tonga study on diabetes, ESRD, BMI, and age; but different on hypothyroidism and RA. Confirmation of the lack of association between diabetes and ESRD with CTS can indicate a wide range of differential diagnoses that can cause CTS-like symptoms in such chronic diseases. This can be a warning that these patients should undergo thorough screening before surgery.
Rhee et al.'s study did not divide patients based on the severity of the disease, but it seems that due to the large sample size of this study, their results on the effect of RA and hypothyroidism on CTS should be considered (1). This difference may be due to the low number of RA and hypothyroid patients in the Tonga and Bahadir's study. Then the interpretation of these results should be done with caution.

\section{AUTHORSHIP CONTRIBUTION}

Study conception and design: YF, BB

Draft manuscript preparation: BB

All authors (FT, SB) reviewed the results and approved the final version of the manuscript.

\section{REFERENCES}

1. Rhee SY, Cho HE, Kim JH, Kim HS: Incidence and reappraisal of known risk factors associated with carpal tunnel syndrome: A nationwide, 11-year, population-based study in south korea. J Clin Neurol 17:524-533, 2021

2. Tonga F, Bahadir S: The factors associated with carpal tunnel syndrome severity. Turk Neurosurg 2021 (Online ahead of print) 